\title{
Cost-Effectiveness Analysis of Anastrozole versus Tamoxifen in Adjuvant Therapy for Early-Stage Breast Cancer - a Health-Economic Analysis Based on the 100-Month Analysis of the ATAC Trial and the German Health System
}

\author{
Michael P. Lux ${ }^{a *}$ Achim Wöckel ${ }^{b *} \quad$ Agnes Benedict $^{c} \quad$ Stefan Buchholz $^{d} \quad$ Noémi Kreif ${ }^{c}$ \\ Nadia Harbeck ${ }^{\text {,f }}$ Rolf Kreienberg ${ }^{b}$ Manfred Kaufmann ${ }^{g}$ Matthias W. Beckmann ${ }^{a}$ \\ Walter Jonat ${ }^{\mathrm{h}}$ Peyman Hadji ${ }^{\mathrm{i}}$ Wolfgang Distler' Guenther Raab ${ }^{\mathrm{j}}$ Hans Tesch' \\ Georg Weyers $^{m}$ Kurt Possinger $^{n} \quad$ Andreas Schneeweiss $^{\circ}$
}

a University Breast Center Franconia, University of Erlangen,

${ }^{\mathrm{b}}$ Department of Gynecology, University of Ulm, Germany

c United BioSource Cooperation, London, UK

${ }^{d}$ Department of Gynecology, University of Regensburg,

e Department of Obstetrics and Gynecology, Technical UniversityMünchen,

${ }^{f}$ Breast Center, University of Cologne,

${ }^{g}$ Centre for Gynecology and Obstetrics, University of Frankfurt,

${ }^{\text {h }}$ Department of Gynecology and Obstetrics, Christian Albrechts University of Kiel,

Department of Gynecology and Gyn-Oncology, Philipps-University of Marburg,

Department of Gynecology and Obstetrics, University of Dresden,

${ }^{k}$ Practice for Gynecology, Munich,

Oncological Practice, Frankfurt,

${ }^{m}$ Practice for Cardiology, Bergisch Gladbach,

n Department of Oncology, Charité Berlin,

- Department of Gynecology and Obstetrics, National Center for Tumor Diseases, University of Heidelberg, Germany

Key Words

Anastrozole $\cdot$ Cost-effectiveness analysis · Cost-utility analysis . Incremental cost-effectiveness ratio - Tamoxifen - QALY

\section{Summary}

Background: In the 'Arimidex', Tamoxifen Alone or in Combination (ATAC) trial, the aromatase inhibitor (AI) anastrozole had a significantly better efficacy and safety profile than tamoxifen as initial adjuvant therapy for hormone receptor-positive $(\mathrm{HR}+)$ early breast cancer $(\mathrm{EBC})$ in postmenopausal patients. To compare the combined long-term clinical and economic benefits, we carried out a cost-effectiveness analysis (CEA) of anastrozole versus tamoxifen based on the data of the 100month analysis of the ATAC trial from the perspective of the German public health insurance. Patients and Methods: A Markov model with a 25-year time horizon was developed using the 100-month analysis of the ATAC trial as well as data obtained from published literature and expert opinion. Results: Adjuvant treatment of EBC with anastrozole achieved an additional 0.32 quality-adjusted life-years (QALYs) gained per patient compared with tamoxifen, at an additional cost of $€ 6819$ per patient. Thus, the incremental cost effectiveness of anastrozole versus tamoxifen at 25 years was $€ 21,069(\$ 30,717)$ per QALY gained. Conclusions: This is the first CEA of an Al that is based on extended follow-up data, taking into account the carryover effect of anastrozole, which maintains the efficacy benefits beyond therapy completion after 5 years. Adjuvant treatment with anastrozole for postmenopausal women with $\mathrm{HR}+\mathrm{EBC}$ is a cost-effective alternative to tamoxifen.

*These two authors contributed equally to this work.
Schlüsselwörter

Anastrozol · Kosten-Effektivitäts-Analyse · Kosten-Nutzwert-Analyse · ICER (Incremental Cost-Effectiveness Ratio) · Tamoxifen · QALY

\section{Zusammenfassung}

Hintergrund: Bei der adjuvanten Therapie von postmenopausalen Patientinnen mit Hormonrezeptor-positivem ( $\mathrm{HR}+$ ) Mammakarzinom belegen die ATAC-100-Monatsdaten (ATAC-Studie: 'Arimidex', Tamoxifen Alone or in Combination) einen signifikanten Vorteil von Anastrozol gegenüber Tamoxifen in Bezug auf Rezidivrisiko und Verträglichkeit. Es wurde eine Kosten-Nutzwert-Analyse von Anastrozol im Vergleich zu Tamoxifen aus der Sicht des deutschen Gesundheitssystems durchgeführt. Material und Methoden: Als Berechnungsbasis wurde ein Markov-Modell zur Abschätzung der Kosteneffektivität entwickelt. Der Modellierungszeitraum umfasste 25 Jahre. Die Daten wurden anhand der ATAC-100-Monatsdaten, vorliegender Literatur und durch ein interdisziplinäres Expertenteam ermittelt. Ergebnisse: Eine adjuvante Therapie mit Anastrozol erzielte 0,32 quality-adjusted life-years (QALYs) pro Patientin mehr, verglichen mit einer adjuvanten Tamoxifentherapie. Die zusätzlichen Kosten der Therapie mit Anastrozol lagen bei $6819 €$ pro Patientin. Im Vergleich mit Tamoxifen erzielte Anastrozol einen ICER (Incremental Cost-Effectiveness Ratio) von $21069 €$ (30 717\$)/QALY über den gesamten Modellierungszeitraum. Schlussfolgerung: Diese KostenNutzwert-Analyse eines Aromatasehemmers basiert erstmals auf einer Datenanalyse, die auch das Follow-Up und den sogenannten Carryover-Effekt nach einer abgeschlossenen 5-Jahres-Therapie beinhaltet. Anastrozol ist auch nach dieser Analyse aus der Sicht des deutschen Gesundheitssystems eine kosteneffektive Therapieoption für postmenopausale Patientinnen mit einem HR+ frühen Mammakarzinom.

\section{KARGER}

Fax +497614520714

Information@Karger.de

www.karger.com
() 2010 S. Karger GmbH, Freiburg

www.karger.com/onk
Dr. Achim Wöckel

Department of Gynecology and Obstetrics

University Hospital Ulm

Pritwitzstraße 43, 89075 Ulm, Germany

Tel. +49 731 500585-01, Fax -02

achim.woeckel@uniklinik-ulm.de 


\section{Introduction}

In Germany, about 57,000 new cases of breast cancer are diagnosed each year, with an overall breast cancer mortality of $26.8 \%$ [1]. Tamoxifen is an established endocrine adjuvant treatment for postmenopausal women with hormone-sensitive breast cancer $[1,2]$. The use of tamoxifen as adjuvant treatment reduces the 5 -year relapse risk by $41 \%$ and the risk of death by $34 \%$ [3]. Furthermore, the risk of contralateral breast cancer is reduced by $39 \%[4,5]$. However, despite these improvements, many women still experience a recurrence and die from the disease. Moreover, tamoxifen has been associated with serious side effects, such as endometrial cancer [6] and thromboembolic disorders [7].

An alternative adjuvant endocrine treatment is anastrozole (Arimidex ${ }^{\circledR}$; AstraZeneca, Wedel, Germany), a third-generation non-steroidal aromatase inhibitor (AI), which has consistently demonstrated superior efficacy and safety results compared to tamoxifen in the 'Arimidex', Tamoxifen Alone or in Combination (ATAC) trial $[8,9]$.

The patient population in the ATAC trial consisted of postmenopausal women with estrogen and/or progesterone receptor-positive early breast cancer (EBC) who had completed primary therapy (surgery \pm radiotherapy \pm chemotherapy) and were eligible for adjuvant endocrine therapy. Patients were randomized to receive either anastrozole or tamoxifen (or both) for up to 5 years [8]. As the combination arm showed no benefit over tamoxifen alone in terms of efficacy or safety, this arm was discontinued following interim analysis at 33 months. Details of the design, methodology and analysis of the trial have been published previously [8]. The present analysis focuses on the results of the two monotherapy arms, anastrozole and tamoxifen, at a median followup of 100 months.

These extended follow-up data showed that, compared to tamoxifen, anastrozole was associated with significantly longer disease-free survival (DFS) (hazard ratio (HR) 0.85, $\mathrm{p}=0.003$ ) and longer time to recurrence (TTR) (HR 0.76, $\mathrm{p}=0.0001$ ) [9]. Recurrence rates for anastrozole-treated patients remained significantly lower after treatment completion (HR 0.75, p = 0.01), indicating a carryover effect of anastrozole that is even greater than that previously shown for tamoxifen [4]. Although overall survival (OS) was statistically not significantly different in the hormone receptorpositive $(\mathrm{HR}+)$ patient group there were numerically fewer deaths (245 vs. 269) after recurrence in the anastrozole group at 100 months [9].

Anastrozole was also associated with a more favorable overall safety profile versus tamoxifen, e.g. a significantly lower incidence of endometrial cancer, thromboembolic events, and vaginal bleeding/discharge. Patients on anastrozole showed increased rates of arthralgia and bone fractures during therapy $[8,10]$; however, no excess of fractures was noted after the 5-year treatment period was completed [9].
The American Society of Clinical Oncology (ASCO) technology assessment has recommended the use of AIs either as initial therapy or after tamoxifen therapy for postmenopausal women with $\mathrm{HR}+\mathrm{EBC}$ as part of the optimal adjuvant hormonal therapy [11, 12]. In Germany, national evidence-based recommendations by the Working Committee of Gynecological Oncology (Arbeitsgemeinschaft Gynäkologische Onkologie $(\mathrm{AGO}))[13]$ and the official consensus guidelines by the German Cancer Society (Deutsche Krebsgesellschaft (DKG)) [14] both recommend the use of AIs as adjuvant endocrine therapy for postmenopausal women with $\mathrm{HR}+\mathrm{EBC}$.

However, the higher acquisition cost of anastrozole, against a background of limited resources in several health systems, could limit the adoption of this agent. Germany and Switzerland, for example, are closely following the USA in terms of health care expenses [15]. Over the coming years, demographic changes including a shift to older patient populations and increasing rates of unemployment are expected to cause increased costs and reduced contributions to the health care systems of many industrialized countries. These developments are likely to have an impact on the availability and reimbursement of new drugs and on medical decision making.

In order to reach fully informed decisions, treatment choices need to be supported by a systematic assessment of the costs and benefits of new health technologies. In many countries, independent institutions, like the National Institute of Clinical Excellence (NICE) in the UK or the Institute for Quality and Efficiency in Health Care (Institut für Qualität und Wirtschaftlichkeit im Gesundheitswesen (IQWiG)) in Germany, have been commissioned to carry out health technology assessments (HTAs) on behalf of the national health care system.

Given the budgetary constraints of many health care systems, it is important to assess if anastrozole offers value for money, i.e. if anastrozole is cost effective compared to the current standard treatment, tamoxifen. The aim of this study was to evaluate the long-term cost effectiveness of anastrozole compared with tamoxifen for postmenopausal women with HR+ EBC. The analysis was carried out from the perspective of the German statutory health insurance (Gesetzliche Krankenversicherung, GKV). It was based on the 100month analysis of the ATAC trial in order to evaluate for the first time the economic impact of the long-term clinical benefits (carryover effect) of anastrozole in upfront treatment.

\section{Methods}

\section{Model Structure}

To assess the cost effectiveness of anastrozole, a probabilistic Markov model was developed [16]. The model follows a hypothetical cohort of 1000 postmenopausal women (mean age 64 years) with HR+ EBC over a 25-year period. A 25 -year time horizon was chosen for the model because of the need to capture the long-term benefits of treatment. A longer time horizon would have had very little impact on the results considering average age at diagnosis and the life expectancy of postmenopausal women 


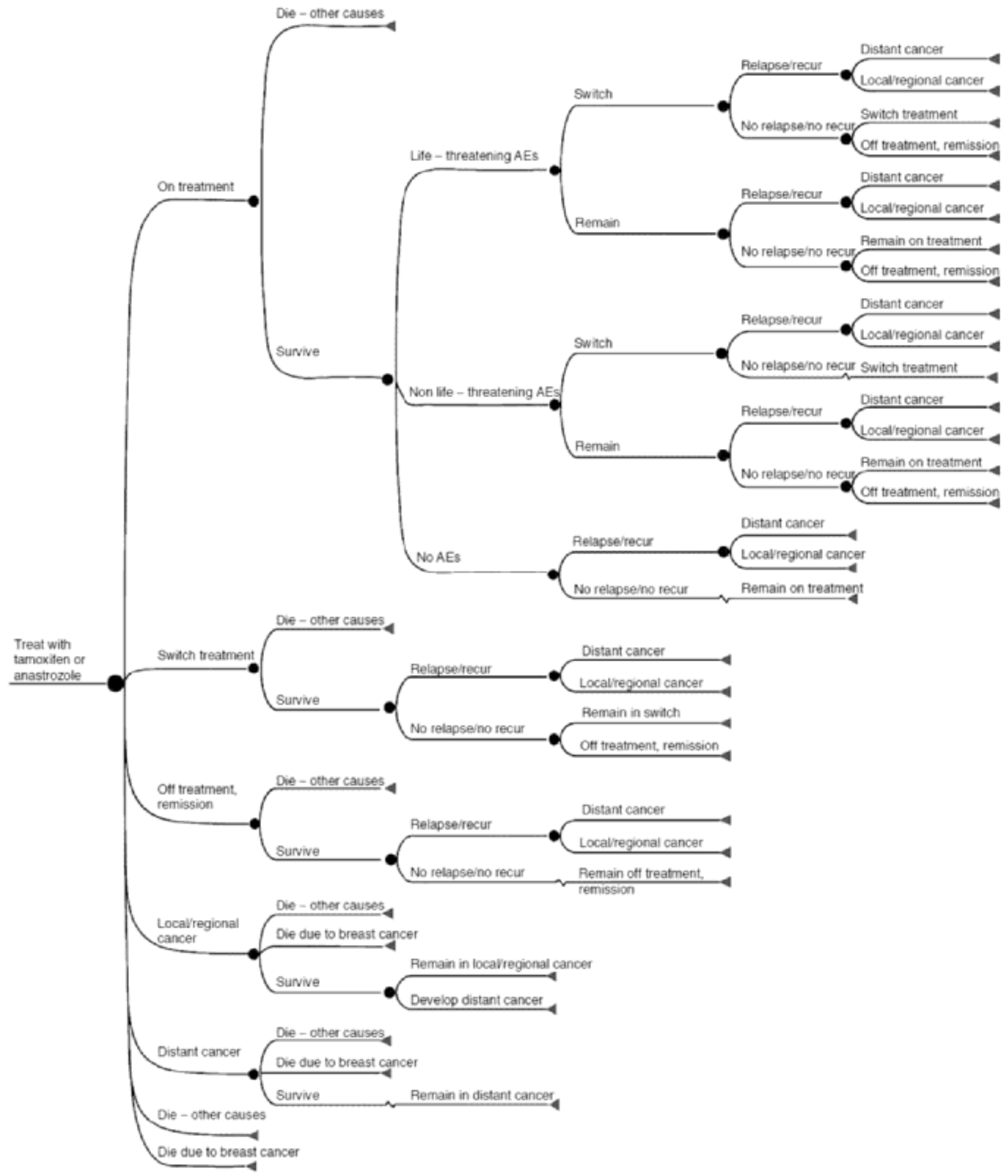

Fig. 1. The Markov decision model for health status.

with EBC, which would lead to the fact that many would already have died within 25 years. In the model, $75 \%$ of the patients will have died by 25 years. The effect of a shorter time horizon is explored in a scenario analysis. Over this time period, patients transition through 7 possible health states at 3-month intervals during the first 5 years (when receiving primary adjuvant treatment of anastrozole or tamoxifen) and at 6-month intervals thereafter (fig. 1). The 7 health states are as follows:

1) on adjuvant endocrine treatment (anastrozole or tamoxifen)

2) on an unplanned switch of adjuvant treatment (e.g. tamoxifen to anastrozole due to side effects) 
Table 1a. Model probabilities: Estimation of Weibull regression parameters for the baseline tamoxifen RFS curve, $1-10$ years $^{\mathrm{a}}$

\begin{tabular}{llll}
\hline Constant (gamma) (SE) & $\begin{array}{l}\text { Logarithm of scale parameter } \\
\text { (lambda) (SE) }\end{array}$ & $\begin{array}{l}\text { Constant/scale } \\
\text { correlation }\end{array}$ & Distribution $^{\mathrm{b}}$ \\
\hline$-3.71085(0.016)$ & $0.0517(0.007)$ & -0.91 & normal \\
\hline
\end{tabular}

${ }^{a}$ Estimates derived using the software STATA 10 SE.

${ }^{b}$ The parameters of the survival regression are normally distributed by the construction of the statistical model.

Table 1b. Model probabilities: TTR hazard ratio for anastrozole vs. tamoxifen [9]

\begin{tabular}{llll}
\hline HR & Lower 95\% CI & Higher 95\% CI & Distribution \\
\hline 0.76 & 0.67 & 0.87 & log-normal \\
\hline
\end{tabular}

Table 1c. Model probabilities: Distant recurrences, withdrawals and deaths

\begin{tabular}{|c|c|c|c|c|c|c|}
\hline & \multicolumn{2}{|c|}{ Distant recurrences, withdrawals and deaths } & \multicolumn{4}{|c|}{ Probabilistic parameters (beta distribution) } \\
\hline & anastrozole, \% & \multirow[t]{2}{*}{ tamoxifen, \% } & \multicolumn{2}{|c|}{ anastrozole } & \multicolumn{2}{|c|}{ tamoxifen } \\
\hline & & & alpha, $\mathrm{n}$ & beta, $\mathrm{n}$ & alpha, n & beta, $\mathrm{n}$ \\
\hline $\begin{array}{l}\text { Distant recurrence (as \% of all recurrences), } \\
\text { years } 1-10[9]^{\mathrm{c}}\end{array}$ & 78.01 & 72.27 & 305 & 86 & 357 & 137 \\
\hline \multicolumn{7}{|l|}{ Adverse events ${ }^{\mathrm{d}}$} \\
\hline Life-threatening & 4.7 & 6.6 & 142 & 2950 & 201 & 2893 \\
\hline Non life-threatening & 69.8 & 65.7 & 2163 & 929 & 2037 & 1057 \\
\hline None & 25.5 & 27.7 & & & & \\
\hline \multicolumn{7}{|l|}{ Withdrawals ${ }^{\mathrm{e}}$} \\
\hline $\begin{array}{l}\% \text { of potentially fatal AEs leading to } \\
\text { withdrawals }\end{array}$ & 26.1 & 25.4 & 37 & 104 & 51 & 105 \\
\hline$\%$ of other AEs leading to withdrawals & 3.3 & 3.5 & 72 & 2163 & 71 & 2037 \\
\hline \multicolumn{7}{|l|}{ Following LRR } \\
\hline Distant metastasis free at 5 years [21] & 52.0 & & 7 & & & 67 \\
\hline Distant metastasis free after 5 years [22] & 77.0 & & 9 & & & 27 \\
\hline Death due to breast cancer after $\mathrm{LRR}^{\mathrm{f}}$ & 22.2 & & 5 & & & 189 \\
\hline \multicolumn{7}{|l|}{ Following DR } \\
\hline Overall survival at 2 years [23] & 50.0 & & 15 & & & \\
\hline
\end{tabular}

${ }^{\mathrm{c}}$ Calculation based on events for $\mathrm{HR}+$ patient in fig. 2: number of distant recurrences divided by number of all first recurrences [9].

${ }^{\mathrm{d}}$ ATAC trial data on file.

'For the purposes of the economic evaluation, the classification of AEs differs from the ones published in the ATAC Trialists' Group, 2008 publication. ${ }^{\mathrm{f}}$ ATAC 5 years trial data on file.

AE, Adverse event; DR, distant recurrence; LRR, local-regional recurrence.

3) off-treatment, in remission

4) with distant recurrence

5) with local/regional recurrence

6) dead due to breast cancer

7) dead due to other causes

All women enter the model at the 'on (adjuvant) treatment' health state when they receive either tamoxifen or anastrozole (fig. 1). At any given time, a patient may either remain in the current health state or make a transition to another state, including death due to breast cancer or other (non-breast cancer) causes. Patients who experience an adverse event (AE)/serious adverse event (SAE) may switch hormonal treatment and transition to the 'on switch of adjuvant treatment' state. In order to simplify the model, it was considered reasonable to allow patients to switch treatment only once. Patients not relapsing will remain on treatment or, after 5 years, transition to the 'off treatment, in remission' state unless they experience a recurrence, which then results in transitioning into the 'distant cancer' or 'local/regional cancer' health states, or die of non-cancer causes. Health outcomes were presented as quality-adjusted life-years (QALYs), the incremental cost-effectiveness ratio (ICER) as cost per QALY gained.

\section{Major Assumptions}

It was assumed that the patient population of the ATAC trial is representative of postmenopausal women with $\mathrm{HR}+\mathrm{EBC}$ in Germany and that the results of the trial apply to the German population. 
Additionally, the analysis is based on the following assumptions:

- The ATAC 100 data showed a carryover effect for anastrozole extending to 9 years beyond treatment initiation. In line with earlier publications and to enable comparisons with previous cost-effectiveness analyses (CEAs) (e.g. Mansel et al. [17]), it was assumed that the carryover effect continued for the period between years 9 and 10. This assumption was based on the continuing divergence of the recurrence curves

Table 2. All-cause annual mortality rates for women in Germany

\begin{tabular}{lcc}
\hline Age cohort & $\begin{array}{l}\text { Non-breast and -endometrial } \\
\text { cancer mortality*, \% }\end{array}$ & $\begin{array}{l}\text { General mortality } \\
\text { rates, \% }\end{array}$ \\
\hline $45-49$ & 0.13 & 0.18 \\
$50-54$ & 0.19 & 0.28 \\
$55-59$ & 0.29 & 0.42 \\
$60-64$ & 0.44 & 0.63 \\
$65-69$ & 0.62 & 0.91 \\
$70-74$ & 1.10 & 1.62 \\
$75-79$ & 2.05 & 3.08 \\
$80-84$ & 3.91 & 6.03 \\
$85-90$ & 7.02 & 10.95 \\
$85-94$ & 14.81 & 22.77 \\
\hline
\end{tabular}

Source: Gesundheitsberichterstattung des Bundes, 2006 [27].

*Also excludes cardiovascular and hip fracture mortality. observed in the completed treatment analysis of the ATAC 100 trial [9] and on the durable treatment benefit previously observed with tamoxifen [3]. However, for a conservative approach, we also included an additional scenario with the same recurrence probabilities for tamoxifen and anastrozole between years 9 and 10 .

- Due to the lack of published data on recurrence rates beyond 10 years, the recurrence rates beyond 10 years are assumed to be the same for both anastrozole and tamoxifen.

- The results of a utility study carried out for the USA [18] are applicable in the German setting.

- Most AEs only occur during the 5-year treatment period. Some serious AEs can occur after this period (i.e. thromboembolic events, endometrial cancer), and the relative risk goes back to the level of the nonbreast cancer patient population after some time (endometrial cancer, thromboembolic events and deep-vein thromboembolism (DVT) after 0.5 years, and hip fracture immediately).

\section{Model Inputs}

\section{Recurrences}

Information on event rates for recurrence and death were derived from the 100-month analysis of the ATAC trial [9]. In order to extrapolate beyond the 100-month follow-up period, parametric survival curves were fitted to both treatment arms. Although there are a number of distributions that can be used to model survival [19], the Weibull distribution is the most common as it does not assume a constant hazard rate and is flex-
Table 3. Costs of medical management and medication

\begin{tabular}{|c|c|c|}
\hline Medical management/medication & Tamoxifen (SD) & Anastrozole (SD) \\
\hline \multicolumn{3}{|l|}{ Medical management } \\
\hline Treatment initiation, $€$ & $397.45(198.72)$ & \\
\hline Routine follow-up first year, $€$ & $211.41(105.71)$ & \\
\hline Routine follow-up 2-5 years, $€$ & $208.14(104.07)$ & \\
\hline Routine follow-up 6+ years, $€$ & $100.72(50.36)$ & \\
\hline Additional monitoring (per 3 months), $€$ & $3.70(1.85)$ & $10.11(5.06)$ \\
\hline \multicolumn{3}{|l|}{ Cost of $\mathrm{AEs}^{\mathrm{a}}$} \\
\hline Non-fatal AEs, $€$ & $592.57(296.29)$ & $752.38(351.19)$ \\
\hline Average AEs (fatal and nonfatal) ${ }^{\mathrm{b}}, €$ & $665.44(332.72)$ & \\
\hline \multicolumn{3}{|l|}{ Off-treatment and recurrence } \\
\hline Off-treatment due to remission (per 3 months), $€$ & $97(47.5)$ & \\
\hline Off-treatment due to AEs (per 3 months), $€$ & $164(84)$ & \\
\hline Diagnosis of LRR recurrence, $€$ & $667(333.5)$ & \\
\hline Diagnosis of DR, $€$ & $570(285)$ & \\
\hline Routine follow-up for LRR (per 3 months), $€$ & $296(148)$ & \\
\hline Routine follow-up for DR (per 3 months) 3 , € & $749(385)$ & $886(443)$ \\
\hline Treatment for $\mathrm{LRR}^{\mathrm{c}}, €$ & $7525(3762.57)$ & $7267(3633.38)$ \\
\hline Treatment for DR (average) $)^{\mathrm{d}}, €$ & $20,190(10,000)$ & \\
\hline \multicolumn{3}{|l|}{ Medication ${ }^{\mathrm{e}}$} \\
\hline Pack price for 100 tablets, $€$ & 22.56 & 572.96 \\
\hline Cost per day, $€$ & 0.23 & 5.73 \\
\hline Cost per cycle, $€$ & 20.53 & 521.39 \\
\hline
\end{tabular}

Source: Calculated using unit costs and physician panel. Unit costs based on EBM2000plus [47] and G-DRG catalog 2008 [48], resource use based on physician expert panel.

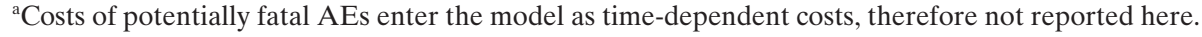

'Used in health state 'switch treatment'.

${ }^{\mathrm{c}}$ Costs differ for the two treatment groups due to the different second-line treatments (tamoxifen for anastrozole patients, anastrozole or letrozole for tamoxifen patients).

${ }^{\mathrm{d}}$ Average cost for treatment of DR, based on estimate from Lux et al. [49] and adjusted for treatment with trastuzumab and/ or lapatinib, assuming a HER2 positivity rate of $11 \%$ in estrogen receptor-positive patients (taken from Witton et al. [50]). ${ }^{\mathrm{e}}$ Rote Liste ${ }^{\circledR} 2008$ [51]. 
Table 4. Utility scores used in the model

\begin{tabular}{|c|c|c|c|c|c|}
\hline \multirow[t]{2}{*}{ Utility item } & \multirow[t]{2}{*}{ Mean (SD) } & \multirow[t]{2}{*}{ Lower CL } & \multirow[t]{2}{*}{ Upper CL } & \multicolumn{2}{|c|}{$\begin{array}{l}\text { Probabilistic parameters } \\
\text { (beta distribution) }\end{array}$} \\
\hline & & & & alpha & beta \\
\hline Disease-free state, no adverse events [18] & $0.965(0.038)$ & 0.954 & 0.976 & 21.61 & 0.78 \\
\hline Common adverse events (tamoxifen) [18] & $0.959(0.043)$ & 0.946 & 0.971 & 19.43 & 0.83 \\
\hline Common adverse events (anastrozole) [18] & $0.958(0.047)$ & 0.944 & 0.971 & 16.49 & 0.72 \\
\hline Vaginal bleeding [18] & $0.926(0.077)$ & 0.903 & 0.948 & 9.78 & 0.78 \\
\hline Endometrial cancer [18] & $0.839(0.23)$ & 0.767 & 0.900 & 1.30 & 0.25 \\
\hline Wrist fracture [18] & $0.852(0.196)$ & 0.793 & 0.905 & 1.94 & 0.34 \\
\hline New contralateral breast cancer [18] & $0.702(0.361)$ & 0.594 & 0.805 & 0.42 & 0.18 \\
\hline Local/regional recurrence [18] & $0.766(0.281)$ & 0.681 & 0.844 & 0.97 & 0.30 \\
\hline Deep-vein thromboembolism [18] & $0.729(0.278)$ & 0.647 & 0.808 & 1.13 & 0.42 \\
\hline Pulmonary embolism [18] & $0.741(0.264)$ & 0.663 & 0.813 & 1.30 & 0.45 \\
\hline Spinal fracture [18] & $0.751(0.229)$ & 0.683 & 0.817 & 1.93 & 0.64 \\
\hline Hip fracture [18] & $0.664(0.309)$ & 0.569 & 0.750 & 0.89 & 0.45 \\
\hline Hormonal therapy for distant recurrence [18] & $0.642(0.319)$ & 0.547 & 0.737 & 0.81 & 0.45 \\
\hline Chemotherapy for distant recurrence [18] & $0.288(0.374)$ & 0.178 & 0.403 & 0.13 & 0.33 \\
\hline Current health $[18]$ & $0.893(0.15)$ & 0.847 & 0.932 & 2.90 & 0.35 \\
\hline Myocardial infarction [32] & $0.750(0.2)$ & NA & NA & 2.77 & 0.92 \\
\hline Stroke [32] & $0.707(0.2)$ & NA & NA & 2.95 & 1.22 \\
\hline Hysterectomy [33] & $0.899(0.23)$ & NA & NA & 0.64 & 0.07 \\
\hline
\end{tabular}

SD, Standard deviation; CL, confidence limit; NA, not applicable.

ible. In addition, Cox HRs can be combined with Weibull survival curves in order to express the treatment effect of an intervention. Weibull distributions can be described with a shape parameter (gamma) and a scale parameter (lambda) [20].

To estimate the parametric survival equation for the tamoxifen arm, the published time-to-first recurrence Kaplan-Meier curve for the tamoxifen arm of ATAC 100 (table 1) was used [9]. The data of the Kaplan-Meier curve were read in using an MS Excel-based statistical application, and the resulting dataset was analyzed with the statistical software STATA10 SE. The Weibull curve estimated for the 100-month follow-up period provided a good fit for the empirical Kaplan-Meier curve. The parameters used in the current model are detailed in table 1a-c.

The parametric survival curve for the anastrozole arm was derived by combining the baseline survival equation for the tamoxifen arm and the published HR of 0.76 (95\% confidence interval (CI): 0.67-0.87) for anastrozole versus tamoxifen [9]. In line with earlier publications and to enable comparisons with previous CEAs (e.g. Mansel et al. [17]), the treatment effect based on the Weibull curves was then extrapolated to 10 years to cover the remaining period between 100 months and 10 years.

After 10 years, a Weibull survival curve fitted to the ATAC TTR data - pooled across the treatment arms - was used to calculate the probability of recurrence for the years 10-25 after initiation of treatment.

Patients who recurred in the model had either a loco-regional recurrence or a distant recurrence, based on the proportion of the two types of recurrence in the ATAC trial, and these were independent of the occurrence of AEs.

Probabilities of distant recurrences among all first recurrences were derived from the 100-month analysis of the ATAC trial [9]. The anastrozole arm had a slightly higher rate of distant recurrences among all first recurrences $(305 / 391=78 \%)$ than the tamoxifen arm $(357 / 494=72 \%)$, but the absolute number of distant recurrences (305 vs. 357) and of all first recurrences ( 391 vs. 494) was higher in the tamoxifen arm. Probabilities of distant metastases and death after local-regional recurrence were taken from the ATAC trial (trial data on file, 60-month data cut) and from the published literature [21-23] (table 1).

\section{Adverse Events, Withdrawals}

The probability of AEs was derived from the ATAC trial $[8,10]$. Key probabilities for $\mathrm{AE}$ and withdrawal rates used in the model, with distributions and sources, are presented in table 1. Based on expert opinion and on observations from ATAC 100, it was assumed that most treatment-related AEs and SAEs occur during the 5-year treatment period. Some events could also occur after completion of treatment (e.g. endometrial cancer and thromboembolic events), and the risk of endometrial cancer and of thromboembolic events was therefore extended for an additional 6 months after treatment completion. All AE rates were assumed to be constant, with the exception of baseline hip fracture rates, where an age-dependent increase was assumed, calculated from data in Icks et al. [24, 25].

The use of AIs is associated with an increased risk of bone loss [26] The use of bone densitometry was not documented in the ATAC trial. Based on expert opinion, some anastrozole patients were assumed to receive initial osteoporosis screening and $5 \%$ of anastrozole patients were assumed to receive bisphosphonates for 5 years. An estimate of bone densitometry usage was also included in the model $(40 \%$ with bone densitometry at treatment initiation, $20 \%$ at 1 st year of routine follow up, $39 \%$ in years $2-5$ and $29 \%$ after year 5 ).

SAEs and AEs could lead to withdrawal. 26.1 and $25.4 \%$ of potentially fatal AEs and 3.3 and $3.5 \%$ of other AEs led to withdrawal, for anastrozole and tamoxifen, respectively. In the model, patients withdrawing from the initial endocrine agent switched to the alternate drug (i.e. patients receiving tamoxifen would be switched to anastrozole, or vice versa). Patients would switch only once, and only between these two drugs.

\section{Mortality}

Equal mortality rates after recurrence were used for each treatment. The probability of death after recurrences was taken from the published literature [23]. All-cause annual mortality (table 2) and general population mortality due to certain SAEs (e.g. endometrial cancer, DVT and ischemic cerebrovascular events) were obtained from national statistics 
Table 5. Projected health outcomes and costs per patient over 25 years (discounted)

\begin{tabular}{lll}
\hline & Tamoxifen & Anastrozole \\
\hline QALYs gained mean $(95 \% \mathrm{CI})$ & $10.05(8.82-11.03)$ & $10.37(9.06-11.38)$ \\
LYs gained mean $(95 \% \mathrm{CI})$ & $11.40(11.29-11.49)$ & $11.69(11.50-11.84)$ \\
RFLY gained mean $(95 \% \mathrm{CI})$ & $10.45(10.35-10.53)$ & $10.88(10.65-11.05)$ \\
Total costs & $€ 16,731(€ 12,937$ to $€ 21,157)$ & $€ 23,550(€ 20,170$ to $€ 27,821)$ \\
Incremental QALYs & & 0.32 \\
Incremental LYs & & 0.29 \\
Incremental RFLYs & & 0.43 \\
Incremental costs & & $€ 6819$ \\
Incremental cost per QALY gained & $€ 21,069(€ 12,567$ to $€ 46,604)$
\end{tabular}

LY, Life-year; QALY, quality-adjusted life-year; RFLY: recurrence-free life-year.

Note: While costs and effects are expressed as rounded values, ICERs are calculated using the exact figures; therefore the ICERs cannot be exactly reconstructed by manual calculation.

Table 6. Scenario analysis

\begin{tabular}{lcccccccc}
\hline & $\begin{array}{l}\text { Base case } \\
\text { results }\end{array}$ & $\begin{array}{l}\text { 10-year time } \\
\text { horizon }\end{array}$ & $\begin{array}{l}\text { 15-year time } \\
\text { horizon }\end{array}$ & $\begin{array}{l}20 \text {-year time } \\
\text { horizon }\end{array}$ & $\begin{array}{l}0 \% \text { discount } \\
\text { rate }\end{array}$ & $\begin{array}{l}5 \% \text { discount } \\
\text { rate }\end{array}$ & $\begin{array}{l}7 \% \text { discount } \\
\text { rate }\end{array}$ & $\begin{array}{l}10 \% \text { discount } \\
\text { rate }\end{array}$ \\
\hline Incremental QALYs & 0.32 & 0.15 & 0.24 & 0.30 & 0.46 & 0.26 & 0.21 & 0.16 \\
Incremental costs, $€$ & 6,819 & 6,483 & 6,623 & 6,748 & 9,754 & 5,650 & 4,798 & 3,879 \\
Cost per QALY gained, $€$ & 21,069 & 44,676 & 27,185 & 22,776 & 21,266 & 21,591 & 22,355 & 23,743 \\
\hline
\end{tabular}

Base case values: time horizon $=25$ years, discount rate $=3 \%$

For variations in the time horizon, the base case value for the discount rate was used, and vice versa.

[27]. Mortality rates due to hip fracture after 5 years, however, were based on age-specific UK data [28] as only one German study was found that only reported 1-year fatality rates $(28.6 \%)$ in older women (average age 76 years) [29].

\section{Resource Utilization and Costs}

Resource utilization was obtained from the literature and an expert panel of 10 office- and hospital-based breast cancer specialists practising in Germany. The data captured management and monitoring of both inpatients and outpatients, including procedures, diagnostic tests, and medications, as well as treatment of SAEs/AEs.

Mean resource use was estimated and unit costs were applied to each resource unit to estimate total costs. Costs were expressed in 2008 Euros. Only direct medical costs were included and were classified into drug costs, costs of recurrences, costs of SAEs and AEs, and follow-up costs (table 3).

For the model, it was assumed that follow-up and surveillance with the recommended diagnostic procedures were performed for 10 years according to current national guidelines [30].

Drug costs were derived from standard sources (table 3). Due to the organizational setup of the health care system in Germany, outpatient and inpatient costs are based on different cost databases (EBM2000plus, G-DRG2008) for inpatient services. Costs were discounted at a 3\% rate per year according to the recent IQWiG guidelines [31].

\section{Calculating Quality-Adjusted Life-Years}

Utility values for the model were obtained from the literature $[18,32,33]$ (table 4).

Utilities for the health states and the majority of SAEs and AEs were based on a cross-sectional study with 44 representative patients with stage I or II operable invasive breast cancer who had previously received 6 months or more of adjuvant hormonal therapy [18]. Utilities for different health states were elicited using a chained standard gamble method, a technique that ascertains patient preference for each health state by estimating the maximum level of risk (of being dead) that an individual is willing to accept in order to avoid each health state [34,35].

The utility scores were assigned to the patients in the corresponding health states in the model and were used to estimate the number of QALYs gained by multiplying them with the number of years patients stayed in that health state. QALYs were discounted by $3 \%$ according to the recent IQWiG guidelines [31].

The uncertainties in applying the utilities to a German population were accounted for in the sensitivity analysis.

\section{Sensitivity Analysis}

In order to explore the uncertainty in the main model inputs, scenario analyses and deterministic and probabilistic sensitivity analyses were carried out.

In the scenario analysis, the effect of the discount rate for cost and benefits was explored in accordance with the IQWiG guidelines [31]. The cost effectiveness results were calculated using a discount rate for cost and benefits of $0,5,7$ and $10 \%$ in addition to the base case scenario of $3 \%$. The effect of different time horizons was also explored, with results reported for 5, 10, 15 and 20 years in addition to the base case scenario of 25 years.

One-way deterministic sensitivity analyses were provided for all major model variables. For the cost and efficacy variables, a range of \pm 1 standard deviation from the mean was used, whereas the $95 \%$ CI was explored for the parameters in the parametric survival equations.

In the probabilistic sensitivity analysis, the incremental cost per QALY gained was calculated using a Monte Carlo simulation of 1000 runs with values drawn from an appropriate probabilistic distribution (see below for further details), resulting in 1000 estimates of expected cost and utility. Results were estimated as means for costs and effects separately, together with an estimate for the $95 \%$ CIs for incremental costs, benefits, and ICERs.

For the probabilistic analysis, distributional assumptions for the different model inputs had to be made. Beta distributions are commonly ac- 
Table 7. One-way sensitivity analysis on incremental costs of anastrozole vs. tamoxifen

\begin{tabular}{llllll}
\hline & \multicolumn{2}{l}{ Input value } & & \multicolumn{2}{l}{ Incremental costs belonging to } \\
\cline { 2 - 6 } & Base case & Low & High & lower value & higher value \\
\hline Cost of routine follow-up for DR, anastrozole, $€$ & 886 & 443 & 1329 & 6286 & 7352 \\
Cost of routine follow-up for DR, tamoxifen, $€$ & 749 & 374 & 1123 & 7327 & 6311 \\
Cost of non-fatal AEs, anastrozole, $€$ & 752 & 376 & 1129 & 6498 & 7140 \\
Cost of non-fatal AEs, tamoxifen, $€$ & 593 & 296 & 889 & 7053 & 6585 \\
Cost of treatment for LRR, tamoxifen, $€$ & 7525 & 3763 & 11,288 & 7009 & 6629 \\
Cost of treatment for DR, $€$ & 20,019 & 10,010 & 30,029 & 6985 & 6653 \\
Cost of treatment for LRR, anastrozole, $€$ & 72.67 & 3633 & 10,900 & 6682 & 6956 \\
Anastrozole HR for recurrence, $€$ & 0.76 & 0.67 & 0.87 & 6720 & 6937 \\
Cost of additional monitoring, anastrozole, $€$ & 10 & 5 & 15 & 6752 & 6886 \\
Cost of off-treatment due to remission, $€$ & 97 & 49 & 146 & 6783 & 6855
\end{tabular}

DR, Distant recurrence; AE, adverse event; LRR, local-regional recurrence; HR, hazard ratio.

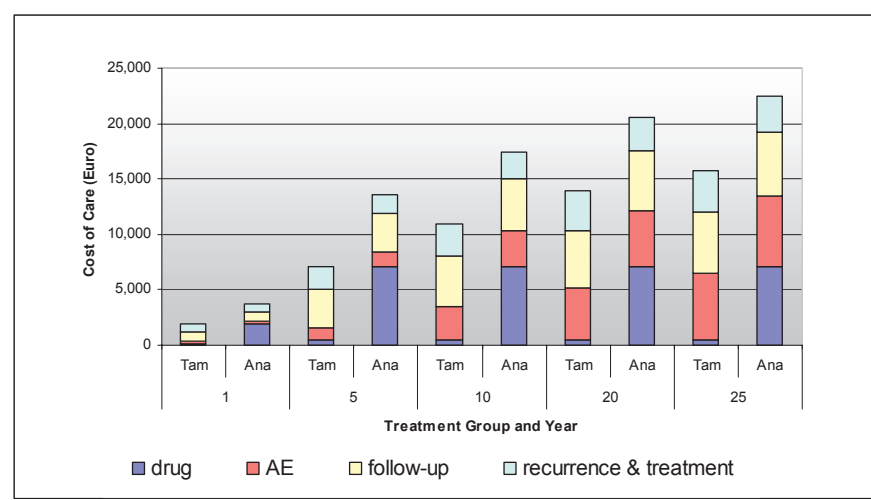

Fig. 2. Total cost of care per patient treated with either tamoxifen (Tam) or anastrozole (Ana) over 5, 10, 20 and 25 years. AE, Adverse event. Note: Drug costs refer only to the trial drugs whereas drugs used in managing recurrences or AEs or terminal care are included under those categories, respectively. End-of-life care is included in the management of recurrences.

cepted to be used to model transition probabilities [36]. Parameters of the beta distributions were calculated based on patient numbers in the trial (table 1). Gamma distributions, based on means and standard deviations, were assumed to be a good fit for skewed and non-negative total cost data for each item (table 3 ). The parameters for the survival regression follow a multivariate normal distribution by assumption, while the HR for anastrozole was drawn from a log-normal distribution. Utility inputs were assumed to follow a beta distribution (table 4). The overall level of uncertainty in the results was expressed as a cost-effectiveness acceptability curve. The curve shows the probability that the ICER lies below a specified willingness to pay (WTP) threshold, i.e. the probability that the intervention is cost effective relative to a given WTP threshold.

\section{Results}

Effectiveness, Costs, and Cost Effectiveness Findings

Over the 25-year time horizon, anastrozole and tamoxifen were associated with mean QALYs of 10.37 and 10.05 per patient, respectively. Anastrozole was also associated with a longer projected (and discounted) overall mean survival

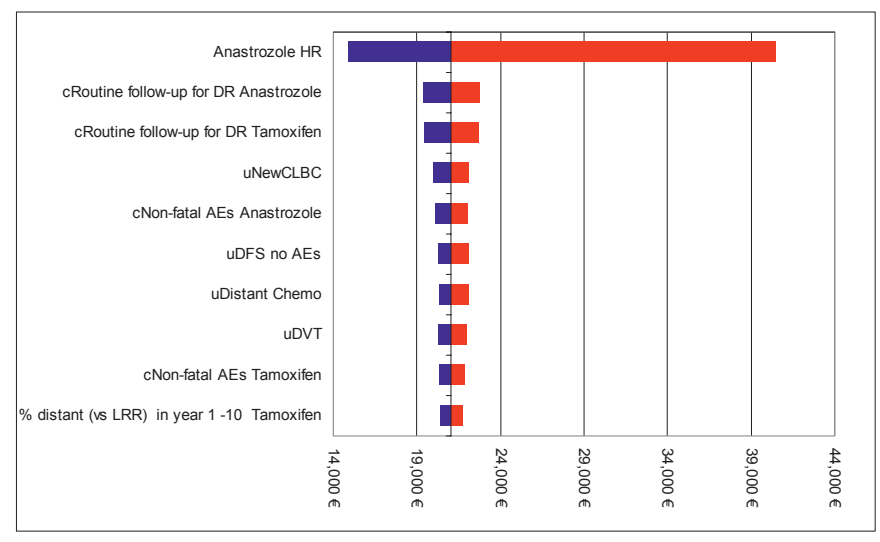

Fig. 3. Tornado chart for incremental cost/QALY for 25 years, anastrozole vs. tamoxifen. 'c' denotes cost inputs, 'u' utility inputs, \% probability inputs; HR: hazard ratio; DR: distant recurrence; LRR: local- regonial recurrence; DFS: disease free survival; AE: adverse event; CLBC: contralateral breast cancer; DVT: deep-vein thromboembolism.

(11.69 versus 11.40 life-years, respectively) and a higher estimated (discounted) cumulative mean cost per patient at 25 years compared with tamoxifen $(€ 23,550(\$ 25,531)$ versus $€ 16,731$ (\$15,826)) (table 5).

Thus, anastrozole was estimated to produce a gain of 0.32 QALYs (or 0.29 life-years survival) at an additional cost of $€ 6819$ (\$9705) per patient over a time horizon of 25 years. The ICER of anastrozole compared with tamoxifen was estimated to be $€ 21,069$ ( $\$ 30,717)$ per QALY gained. The incremental cost per life-year gained was $€ 23,412$ (\$37,867). An additional scenario was run when for years 9 and 10 the same recurrence probabilities were used for tamoxifen and anastrozole. This modification did not substantially alter the results (ICER: €24,319/QALY).

\section{Sensitivity Analysis}

The scenario analysis for different discount rates (table 6) showed that the incremental cost per QALY results changed only moderately when altering the discount rates for costs and 
Table 8. One-way sensitivity analysis on incremental QALYs of anastrozole vs. tamoxifen

\begin{tabular}{lllll}
\hline & \multicolumn{2}{l}{ Input value } & & \multicolumn{2}{c}{ Incremental benefit belonging to } \\
\cline { 2 - 5 } & Base case & Low & High & lower value \\
\hline Anastrozole HR & 0.76 & 0.67 & 0.87 & 0.45 \\
Utility of new CLBC & 0.70 & 0.34 & 1.00 & 0.17 \\
Utility of DFS no AEs & 0.97 & 0.93 & 1.00 & 0.34 \\
Utility of DVT & 0.73 & 0.45 & 1.00 & 0.31 \\
Utility of distant chemo & 0.29 & 0.00 & 0.66 & 0.31 \\
\% distant (vs. LRR) in years 1-10, tamoxifen & 0.72 & 0.70 & 0.74 & 0.34 \\
Utility of common AEs anastrozole & 0.96 & 0.91 & 1.00 & 0.31 \\
\% distant (vs LRR) in years 1-10, anastrozole & 0.78 & 0.76 & 0.80 & 0.31 \\
Utility of LRR & 0.77 & 0.49 & 1.00 & 0.31 \\
Utility of common AEs, tamoxifen & 0.96 & 0.92 & 0.33 \\
\hline
\end{tabular}

HR, Hazard ratio; CLBC, contralateral breast cancer; DFS, disease-free survival; AE, adverse event; DVT, deep-vein thromboembolism; DR, distant recurrence; LRR, local-regional recurrence.
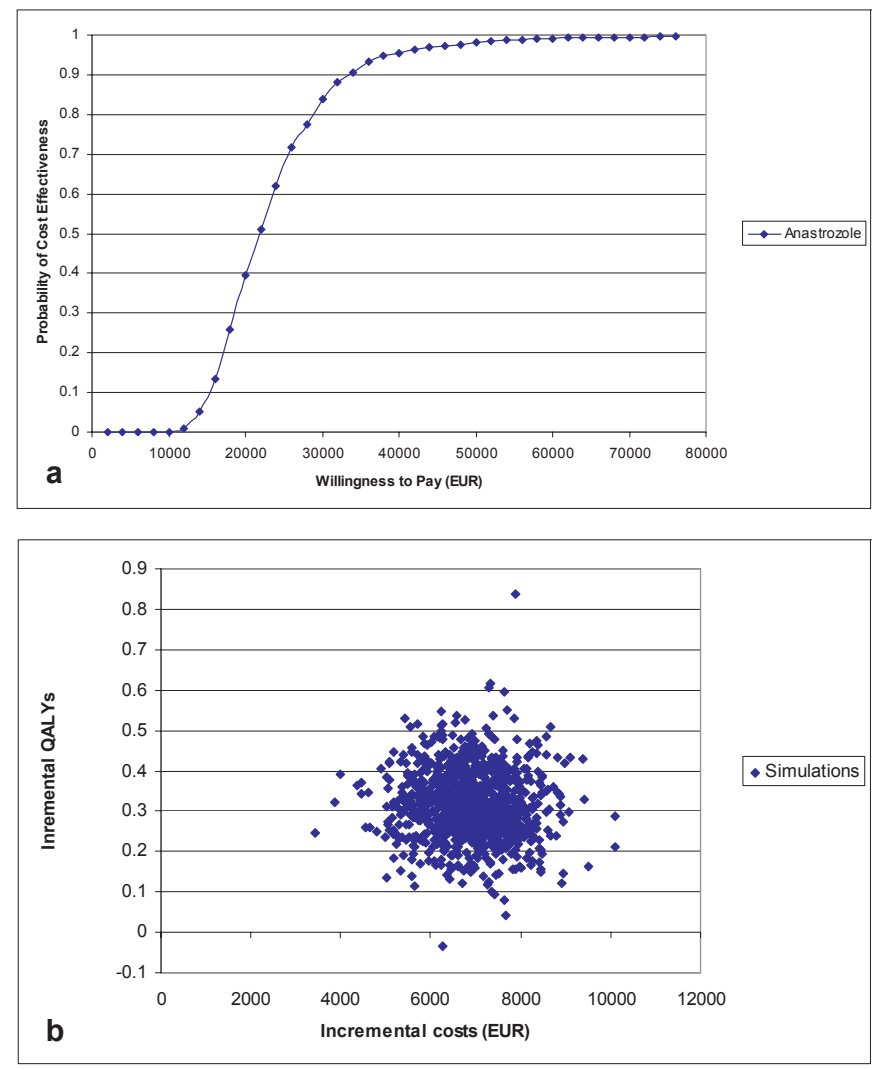

Fig. 4. Probabilistic results. a Cost-effectiveness acceptability curve for anastrozole (25 years). b Cost-effectiveness plane, anastrozole vs. tamoxifen, incremental cost/QALY gained. Note: Based on a Monte Carlo simulation of 1000 runs.

benefits. Using a $10 \%$ discount rate instead of the $3 \%$ rate used in the base case, the ICER increased by $17 \%$. Changing the time horizon had a substantial effect on the results (table 6): While benefits are accrued at a relatively steady rate over the entire lifetime, the majority of the costs arise within the first 5 years of treatment (fig. 2), leading to a decrease in the ICER over longer time horizons.
The results of the deterministic one-way sensitivity analysis are represented by a tornado chart (fig. 3). The single most important model input is the HR for recurrence-free survival of anastrozole over tamoxifen. Varying this input from 0.67 to 0.87 (the base case value is 0.76 ) decreased the ICER to below $€ 15,000 / \mathrm{QALY}(\$ 22,032)$ for 0.67 and above $€ 40,000 / \mathrm{QALY}$ $(\$ 58,752)$ for 0.87 . The effects of the most powerful inputs on incremental costs and benefits are shown in tables 7 and 8 , respectively.

The results of the probabilistic sensitivity analysis using a Monte Carlo simulation of 1000 runs of the model are shown in figure 4 . The resulting cost-effectiveness acceptability curve (fig. 4) indicated a greater than $90 \%$ probability that the cost per QALY gained with anastrozole would be less than $€ 30,000(\$ 34,867)$ and a $50 \%$ probability that it would be less than $€ 20,000(\$ 29,376)$. The ICER had a $95 \%$ non-parametric CI of $€ 12,567$ to $€ 46,604$ per QALY gained $(\$ 18,458$ to $\$ 68,451$ ) as reflected in the acceptability curve (fig. 4 ).

\section{Discussion}

This study is the first CEA of an AI that is based on extended follow-up data, taking into account the carryover effect of anastrozole, which maintains the efficacy benefits beyond the completion of treatment [9].

In the current analysis, the use of anastrozole compared to tamoxifen as adjuvant therapy in postmenopausal women with $\mathrm{HR}+\mathrm{EBC}$ results in an improvement in survival (290 years gained) and quality of life (320 QALYs gained) in a cohort of 1000 patients over a period of 25 years. For the individual patient, the benefits were provided at an incremental cost of $€ 21,069$ per QALY gained (95\% CI: $€ 12,567$ to $€ 46,604)$.

The available long-term clinical data do not show statistically significant OS gains with AIs [9, 37], and this has been discussed controversially [38, 39]. Seruga and Tannock argue that DFS is an imperfect surrogate for OS in adjuvant trials 
for breast cancer. They maintain that, because of this lack of improvement in OS, the results of cost-benefit analyses in AIs are unreliable [38]. On the other hand, Cuzick argues that DFS is a well-accepted endpoint in adjuvant studies of breast cancer and other good-prognosis cancers [39]. OS can no longer be regarded as the primary endpoint for trials in which the prognosis is good, because of the large dilutional effect of death from other causes. Furthermore, the time from distant recurrence to death in patients with breast cancer is commonly 2-3 years and longer, so that a longer follow-up time will be needed to accrue enough events to provide similar power to that achievable using distant recurrence. The Early Breast Cancer Trialists' Collaborative Group analyzed the 5 -year and 10-year effects of various systemic adjuvant therapies on breast cancer recurrence and survival [40]. While the effect on recurrence is seen during the first 5 years, most of the effect on breast cancer mortality comes after this period [40]. OS can be improved, but treatments normally have relatively small effects on mortality from other causes, which is usually high in older patients (the typical population in the large AI trials).

Our analysis, consistent with the clinical trial results, did not assume any OS benefit and showed non-parametric CIs just overlapping at the 10-year time horizon (95\% CI: tamoxifen: 7.69-7.76 life-years gained (LYG); anastrozole: 7.76-7.86 LYG) around the OS estimates for the two treatment arms. However, over 25 years, a numerically longer survival is achieved by a longer TTR for patients on anastrozole. In this analysis, it is the improvement in recurrence-free survival and the avoidance of therapy costs for local recurrence and distant metastasis that underlie the cost-effectiveness ratio in the adjuvant setting.

The use of a Markov model required several assumptions which require justification. First, a 25-year time horizon was chosen in order to consider the long-term benefits of treatment, including the carryover effect. This time horizon also implies that by the end of the 25-year time frame most patients in the model would have died. Given that a substantial proportion of the costs accrues within the initial 5-year treatment period (e.g. drug acquisition costs, etc.) whereas the survival effects occur for at least another four years based on the current data [9], the ICER of $€ 21,069$ per QALY gained would be lower over longer time horizons and higher over shorter time horizons. To test for the effect of assuming a carryover effect extending beyond the trial time horizon, an additional scenario was run where anastrozole did not have a recurrence benefit compared to tamoxifen in years 9 and 10 . The resulting ICER ( $€ 24,319$ per QALY) was not substantially higher than the base case result.

Another potential limitation is that some inputs for the model (e.g., estimates for resource use, definition of treatment, etc.) are based on clinical expert opinion. A better source of information would have been a national database linked to diagnosis or resource use data collected alongside clinical trials on adjuvant endocrine therapy in EBC. In the foreseeable future, CEAs of therapeutic interventions are likely to become an integral part of health care policy in Germany as well as other countries. As research into health-economical efficiency of new drugs will become increasingly important, evaluation of economical data as secondary outcomes should be considered in the design of clinical studies.

The model also assumed that AEs/SAEs only occurred over the initial 5-year treatment period, with the exception of endometrial cancer and thromboembolic events. Thus, the model treated AEs/SAEs separately in order to account for the potentially increased mortality and their effect beyond treatment.

Several studies analyzing the cost effectiveness of anastrozole compared to tamoxifen were identified. All these analyses, however, used the 5-year completed treatment analysis data from the ATAC trial [17, 41-43]. Karnon [44] performed a review of several published health economic evaluations of AIs in the adjuvant setting. He identified 15 separate studies that evaluated AIs. Due to the comparatively long clinical experience and the amount of data available, CEAs of anastrozole have been studied extensively. The combined results from studies using a range of methodological approaches suggest that the use of AIs, including anastrozole, as adjuvant endocrine treatment for EBC is a cost-effective alternative to current standard therapies, although their effectiveness for very-low-risk tumors remains uncertain. The study by Locker et al. evaluated the cost effectiveness of anastrozole versus tamoxifen for primary adjuvant treatment from the US health care perspective [43]. The incremental cost effectiveness for anastrozole versus tamoxifen was $\$ 20,246$ per QALY gained (equivalent to $€ 15,387$, based on an exchange rate of $\$ 1=$ $€ 0.76$, October 2008) and thus within the acceptable reimbursement range in the US $(\$ 50,000$ to $\$ 100,000$ per QALY gained). In contrast, based on 47 months of trial data, the Hillner study found a much higher incremental cost effectiveness for anastrozole at $\$ 75,900$ per QALY gained (equivalent to $€ 33,924$; exchange rate of $\$ 1=€ 0.76$, October 2008) [42]. A wide range of one-way sensitivity analyses were performed which show that the results were sensitive to the risk of hip fracture. The model assumed an OR of 1.6 for hip fractures during anastrozole therapy, which is higher than the OR used in later analyses (OR 1.17). This leads to higher resource costs by treatment of fractures. Other differences from the current study include the choice of time horizon (20 years), utility values applied, resource use and handling of AEs. However, the main reason for the different results might well be the different HR for TTR, a parameter that was shown in our sensitivity analysis to be the single most important model input. Whereas Hillner et al. [42] used the TTR HR that was available at the time $(\mathrm{HR}=0.79)[8]$, the current model uses the TTR HR from the 100-month analysis $(\mathrm{HR}=0.76)$ [9]. The resulting advantage in recurrence-free survival would therefore lead to lower ICERs. 
A more direct comparison can be made with the study of Mansel et al. [17], who carried out a UK adaptation of the current model using the same time horizon but a different base case discount rate of $3.5 \%$ (as recommended by NICE). In that study, using the UK National Health Service (NHS) perspective, the ICER of anastrozole compared with tamoxifen was $£ 17,656$ per QALY gained, which is within NICE's accepted threshold of $£ 20,000$ to $£ 30,000$ per QALY. This is equivalent to an incremental cost of $€ 22,728$ per QALY gained (exchange rate $£ 1=€ 1.28$, October 2008), which is similar to that found in the present study (€20,913 per QALY gained). Skedgel and colleagues analyzed the cost effectiveness of anastrozole from a Belgian perspective and reported a cost of $€ 19,992$ per QALY gained [45].

Some studies have attempted to evaluate the impact of a carryover effect on the overall results of a CEA. In the study by Mansel et al., it was assumed that a carryover effect persists for a further 5 years after treatment completion [17]. Karnon et al. [41] analyzed the ICER of anastrozole versus tamoxifen with and without a carryover effect of the AI. Without the effect, an ICER of $£ 11,428$ (95\% CI: $£ 6131$ to $£ 53,125$ ) was reported. When the carryover effect was included, the ICER decreased to $£ 7,015$ (95\% CI: $£ 3316$ to $£ 31,997$ ), lower than the result of the present German study. A likely explanation for this difference is that Karnon et al. based their calculation on extrapolating the more favorable HR from the 5-year ATAC data $(\mathrm{HR}=0.74)$, which resulted in larger gains in life-years and QALYs than those obtained from using the published data of the 100-month analysis (life-years gained: 0.35 vs. 0.29 in the present study; QALYs gained: 0.36 vs. 0.32 in the present study).

In Germany, cost-effectiveness ratios are recognized by IQWiG as an important tool to aid decision making. HTAs by the IQWiG are likely to become a requirement for decisions regarding the reimbursement of new medicines. However, there is as yet no fixed WTP threshold for ICERs above which a technology would automatically be defined as not cost effective; therefore, a definitive interpretation about cost-effectiveness ratios found in this study cannot be made. It was felt that a CEA of anastrozole from the perspective of the German health care system was desirable for a number of reasons.
Firstly, there are differences between the German health care system (e.g. general organizational structure, costs, resource use, etc.) and those of other countries for which CEAs for anastrozole have been performed [46]. Secondly, the creation of a the German health technology assessment institute (IQWiG) with its mandate to perform HTAs for new health care interventions requires national CEAs that are based on national data. The results of the current model and of previous cost effectiveness studies from other countries suggest that the underlying benefits of anastrozole in the treatment of postmenopausal women with $\mathrm{ER}+\mathrm{EBC}$ translate into a cost effectiveness that is largely consistent among different health care systems. A comparison of the cost effectiveness of different AIs based on clinical data from a direct comparison (e.g. anastrozole and letrozole in the ongoing Femara versus Anastrozole Clinical Evaluation (FACE) trial) would be desirable in order to gain further insight into the cost effectiveness of AIs in patients with $\mathrm{HR}+\mathrm{EBC}$.

\section{Conclusions}

Anastrozole has been demonstrated to be clinically effective and well tolerated by postmenopausal women with $\mathrm{HR}+\mathrm{EBC}$ undergoing adjuvant endocrine therapy. The current study shows that, based on long-term follow-up data, it represents a cost-effective alternative to tamoxifen for this patient population in Germany.

\section{Acknowledgement}

We are grateful to all patients who participated in the utility studies.

\section{Conflict of Interest}

This study was supported by an unrestricted grant from AstraZeneca, Germany. The authors have received honoraria from AstraZeneca for consultation. Moreover, they have received travel expenses for meetings to prepare this analysis.

\section{References}

1 Robert Koch Institut: Brustdrüse der Frau; in Robert Koch-Institut und Gesellschaft der epidemiologischen Krebsregister in Deutschland e.V. (Hrsg): Krebs in Deutschland 2003-2004. Häufigkeiten und Trends, ed 6. Berlin, Westkreuz-Druckerei 2008, pp 54-58.

2 Early Breast Cancer Trialists' Collaborative Group: Tamoxifen for early breast cancer: an overview of the randomised trials. Lancet 1998;351: 1451-1467.

3 Early Breast Cancer Trialists' Collaborative Group: Tamoxifen for early breast cancer. Cochrane Database Syst Rev 2001;1:CD000486.
4 Early Breast Cancer Trialists' Collaborative Group: Effects of chemotherapy and hormonal therapy for early breast cancer on recurrence and 15 -year survival: an overview of the randomised trials. Lancet 2005;365:1687-1717.

5 Fisher B, Costantino J, Redmond C: A randomized clinical trial evaluating tamoxifen in the treatment of patients with node-negative breast cancer who have estrogen-receptor-positive tumors. N Engl J Med 1989;320:479-484.
6 Fisher B, Dignam J, Bryant J, Wolmark N: Five versus more than five years of tamoxifen for lymph node-negative breast cancer: updated findings from the National Surgical Adjuvant Breast and Bowel Project B-14 randomized trial. J Natl Cancer Inst 2001;93:684-690.

7 Love RR, Wiebe DA, Newcomb PA: Effects of tamoxifen on cardiovascular risk factors in postmenopausal women. Ann Intern Med 1991;115: 860-864.

8 ATAC Trialists' Group: Results of the ATAC (Arimidex, Tamoxifen Alone or in Combination) trial after completion of 5 years' adjuvant treatment for breast cancer. Lancet 2005;365:60-62. 
9 Arimidex, Tamoxifen, Alone or in Combination (ATAC) Trialists' Group; Forbes JF, Cuzick J, Buzdar A: Effect of anastrozole and tamoxifen as adjuvant treatment for early stage breast cancer: 100 month analysis of the ATAC trial. Lancet Oncol 2008;9:45-53.

10 The Arimidex, Tamoxifen, Alone or in Combination Trialists' Group, Buzdar A, Howell A, Cuzick J: Comprehensive side-effect profile of anastrozole and tamoxifen as adjuvant treatment for earlystage breast cancer: long-term safety analysis of the ATAC trial. Lancet Oncol 2006;8:633-643.

11 Winer EP, Hudis C, Burstein HJ: American Society of Clinical Oncology technology assessment on the use of aromatase inhibitors as adjuvant therapy for postmenopausal women with hormone receptor-positive breast cancer: status report 2004. J Clin Oncol 2005;23:619-629.

12 Goldhirsch A, Coates AS, Gelber RD; St Gallen Expert Panel Members: First-select the target: better choice of adjuvant treatments for breast cancer patients. Ann Oncol 2006;17:1772-1776.

13 Arbeitsgemeinschaft Gynäkologische Onkologie (AGO): www.ago-online.de/download/ g_mamma_08_1_1_c_02_adj_endo_therapy_ postmenopausal patients.pdf, 2009.

14 Kreienberg R, Kopp I, Albert U, et al.: Interdiszplinäre S3-Leitlinie - Diagnostik, Therapie und Nachsorge beim Mammakarzinom. Munich, Zuckschwerdt Verlag, 2008.

15 Lux MP, Beckmann MW: Klinische Ökonomie in der gynäkologischen Onkologie. Frauenheilkunde aktuell 2008;2:35-41.

16 Sonnenberg FA, Beck JR: Markov models in medical decision making: a practical guide. Med Decis Making 1993;13:322-338.

17 Mansel R, Locker G, Fallowfield L: Cost-effectiveness analysis of anastrozole vs tamoxifen in adjuvant therapy for early stage breast cancer in the United Kingdom: the 5 year completed treatment analysis of the ATAC trial. Br J Cancer 2007;97: 152-161.

18 Sorensen SV, Brown R, Benedict A: Patient-rated utilities in postmenopausal early breast cancer (EBC): a cross-country comparison. Value Health 2004;7:641-642.

19 Lee ET: Statistical methods for survival data analysis, 2 ed. New York, John Wiley and Sons, 1992.

20 Kleinbaum D, Klein M: Survival analysis: a selflearning text. Berlin, Springer, 2005.

21 Kamby C, Sengelov L: Pattern of dissemination and survival following isolated locoregional recurrence of breast cancer. Breast Cancer Res Treat 1997;45:181-192.

22 Moran M, Haffty B: Local-regional breast cance recurrence: prognostic groups based on patterns of failure. Breast J 2002;8:81-87.
23 Stockler M, Wilcken NR, Ghersi D, Simes RJ: Systematic reviews of chemotherapy and endocrine therapy in metastatic breast cancer. Cancer Treat Rev 2000;26:151-168.

24 Icks A, Haastert B, Wildner M: Trend of hip fracture incidence in Germany 1995-2004: a populationbased study. Osteoporos Int 2004;19:1139-1145.

25 Icks A, Haastert B, Wildner M: Hip fracture incidence in Germany: analysis of the national hospital discharge registry. Dtsch Med Wochenschr 2008; 133:125-128.

26 Shapiro CL: Aromatase inhibitors and bone loss: risk in perspective. J Clin Oncol 2005;23: 4847-4849.

27 Gesundheitsberichterstattung des Bundes, www. gbe-bund.de, 2006.

28 Roberts SE, Goldacre MJ: Time trends and demography of mortality after fractured neck of femur in an English population, 1968-98: database study. BMJ 2003:327:771-775.

29 Raunest J, Engelmann R, Jonas M: Morbidität und Letalität bei hüftgelenknahen Femurfrakturen im höheren Lebensalter. Ergebnisse einer prospektiven Studie. Unfallchirurg 2001;104:325-332.

30 Beckmann MW, von Minkwitz G, Pfisterer J: Stellungnahme der Arbeitsgemeinschaft Gynäkologische Onkologie (AGO). Nachsorge beim Mammakarzinom und bei gynäkologischen Malignomen - 'To follow-up or not to follow-up' Follow-up Care After Breast Cancer and Gynecologic Malignancies: Position Statement of the German Gynecologic Oncology Working Group. Geburtsh Frauenheilk 2003;63:725-730.

31 Institut für Qualität und Wissenschaftlichkeit im Gesundheitswesen: Methods for assessment of the relation of benefits to costs in the German statutory health care system. Version 1.1. http://iqwig.de/ index.805.en.html, 2008.

32 Tengs TQ, Wallace A: One thousand health-related quality of life estimates. Med Care 2000;38: 583-637.

33 Sculpher M, Manca A, Abbott J: Cost effectiveness analysis of laparoscopic hysterectomy compared with standard hysterectomy: results from a randomised trial. BMJ 2004;328:134

34 Torrance GW: Measurement of health state utilities for economic appraisal: a review. J Health Econ 1986;5:1-30.

35 Bennett KJ, Torrance GW: Measuring health state preferences and utilities: rating scale, time tradeoff and standard gamble techniques; in Spilker B (ed): Quality of life and pharmacoeconomics in clinical trials, ed 2. Philadelphia, Lippincott-Raven 1996, pp 253-265.

36 Briggs A: Handling uncertainty in economic evaluations and presenting the results; in Drummond $\mathrm{M}$, McGuire AM (eds): Economic evaluation in health care: merging theory with practice. Oxford, Oxford University Press, 2001, pp 172-214.
37 Koeberle D, Thuerlimann B: Letrozole as upfront endocrine therapy for postmenopausal women with hormone-sensitive breast cancer: BIG 1-98. Breast Cancer Res Treat 2007;105(suppl 1):55-66.

38 Seruga B, Tannock IF: Up-front use of aromatase inhibitors as adjuvant therapy for breast cancer: the emperor has no clothes. J Clin Oncol 2009;27: 840-842.

39 Cuzick J: Primary endpoints for randomised trials of cancer therapy. Lancet 2008;371: 2156-2158.

40 Early Breast Cancer Trialists' Collaborative Group (EBCTCG): Effects of chemotherapy and hormonal therapy for early breast cancer on recurrence and 15-year survival: an overview of the randomised trials. Lancet 2005;365:1687-1717.

41 Karnon J, Delea T, Barghout V: Cost utility analysis of early adjuvant letrozole or anastrozole versus tamoxifen in postmenopausal women with early invasive breast cancer: the UK perspective. Eur J Health Econ 2008;9:171-183.

42 Hillner BE: Benefit and projected cost-effectiveness of anastrozole versus tamoxifen as initial adjuvant therapy for patients with early-stage estrogen receptor-positive breast cancer. Cancer 2004;101: 1311-1322.

43 Locker GY, Mansel R, Cella D: Cost-effectiveness analysis of anastrazole versus tamoxifen as primary adjuvant therapy for postmenopausal women with early breast cancer: a US healthcare system perspective. 5-Year completed treatment analysis of the ATAC ('Arimidex' Tamoxifen Alone or in Combination) trial. Breast Cancer Res Treat 2008; 106:229-238.

44 Karnon J: Cost-effectiveness of letrozole, anastrozole and exemestane for early adjuvant breast cancer. Expert Rev Pharmacoecon Outcomes Res 2007;7:143-153.

45 Skedgel C, Rayson D, Dewar R, Younis T: Costutility of adjuvant hormone therapies with aromatase inhibitors in post-menopausal women with breast cancer: upfront anastrozole, sequential tamoxifen-exemestane and extended tamoxifenletrozole. Breast 2007;16:252-261.

46 Hind D, Ward S, De Nigris E: Hormonal therapies for early breast cancer: systematic review and economic evaluation. Health Technol Assess 2007;11: $1-152$.

47 EBM 2000plus, www.kbv.de/8144.html, 2008.

48 G-DRG2008, www.g-drg.de, 2008

49 Lux MP, Hartmann M, Jackisch C, et al.: Cost-utility analysis for advanced breast cancer therapy in Germany: results of the fulvestrant sequencing model. Breast Cancer Res Treat 2009;117:305-317.

50 Witton CJ, Reeves JR, Going JJ, Cooke TG, Bartlett JM: Expression of the HER1-4 family of receptor tyrosine kinases in breast cancer. J Pathol 2003;200:290-297.

51 Rote Liste, www.rote-liste.de, 2008. 